\title{
A scanning transmission $x$-ray microscope for materials science spectromicroscopy at the advanced light source
}

T. Warwick, K. Franck, J. B. Kortright, G. Meigs, M. Moronne, S. Myneni, E. Rotenberg,

S. Seal, and W. F. Steele

Lawrence Berkeley National Laboratory, University of California, Berkeley, California 94720

H. Ade and A. Garcia

Department of Physics, North Carolina State University, Raleigh, North Carolina 27695

S. Cerasari

Universitá di Trieste, 134127 Trieste, Italy

J. Denlinger

University of Michigan, Ann Arbor, Michigan 48109

S. Hayakawa

School of Engineering, University of Tokyo, Tokyo 113, Japan

A. P. Hitchcock and T. Tyliszczak

Department of Chemistry, McMaster University, Hamilton, Ontario L8S 4M1, Canada

J. Kikuma

ASAHI Chemical Industry Co., Fuji shi 416, Japan

E. G. Rightor

DOW Chemical, Midland, Michigan 48641

H.-J. Shin

Pohang Accelerator Laboratory, POSTECH, Pohang 790784, Korea

B. P. Tonner

Department of Physics, University of Wisconsin, Milwaukee, Wisconsin 53211

(Received 23 April 1998; accepted for publication 26 May 1998)

Design and performance of a scanning transmission X-ray microscope (STXM) at the Advanced Light Source is described. This instrument makes use of a high brightness undulator beamline and extends the STXM technique to new areas of research. After 2.5 years of development it is now an operational tool for research in polymer science, environmental chemistry, and magnetic materials. (C) 1998 American Institute of Physics. [S0034-6748(98)04908-9]

\section{INTRODUCTION}

Scanning transmission x-ray microscopy (STXM) generates microscopic images of a thin section of specimen by raster-scanning in a focused x-ray beam. The flux of transmitted $x$ rays is measured to obtain the image intensity. This technique has been developed ${ }^{1,2}$ and applied to the life sciences. ${ }^{3} \mathrm{X}$-ray spots close to the diffraction limit have been reported. ${ }^{2,4} \mathrm{X}$-ray absorption spectra can be obtained from microscopic features on the sectioned sample. ${ }^{5}$ This technique falls into the category of 'spectromicroscopy' and finds application to problems in materials science, where $\mathrm{x}$ ray absorption spectra can be characteristic of the chemical state of atomic species and the structure of crystalline materials. ${ }^{6}$

The best spatial resolution in focused x-ray microscopy is presently achieved when Fresnel zone plates are used as the focusing elements. ${ }^{7}$ Coherently illuminated zone plate microscopes typically operate near their diffraction limit. The phase space acceptance for photons is then small (of the order $\lambda^{2}$ ). The intensity of the signal is proportional to the brightness of the illumination, so that an undulator beamline at a third generation synchrotron radiation source is an attractive place to develop these instruments.

We have implemented a pair of scanning zone plate microscopes at the Advanced Light Source, on undulator beamline 7.0. This article reports the initial performance of the first of these two.

Figure 1 shows the tandem layout of the two microscopes. The upstream microscope is an ultrahigh vacuum (UHV) scanning photoelectron zone plate microscope (SPEM) which is being commissioned. The STXM is the last item in the beamline. It has been in operation for about 1 year, during which time we have been developing the software control, and learning how to use it in the beamline, which also serves spectroscopy experiments on another branch.

There are three capabilities we want to exploit in this STXM; areas where this $\mathrm{x}$-ray technique has unique strengths.

\section{A. Radiation damage to the sample can be minimal}

Near-edge x-ray absorption fine-structure spectroscopy (NEXAFS) and electron energy loss spectroscopy (EELS) 


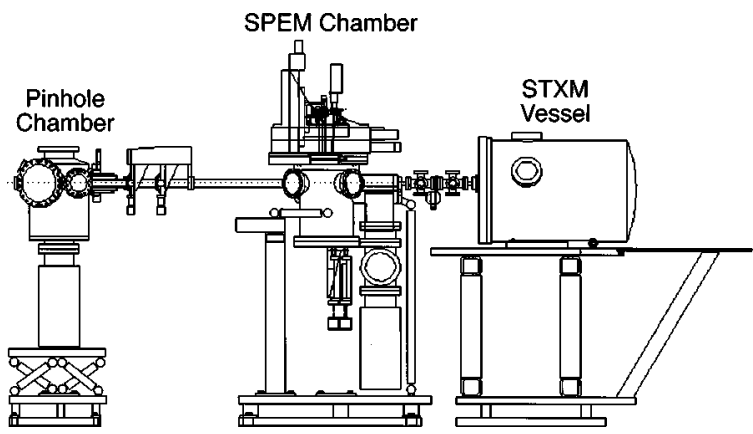

FIG. 1. Tandem scanning zone plate microscopes at beamline 7.0. The beam passes through the pinhole (optional) and illuminates the scanning photoelectron microscope (SPEM) which operates in UHV, or passes on through a silicon nitride window, into the helium environment of the scanning transmission X-ray microscope (STXM) at the end of the beamline.

are two techniques to obtain the cross section for exciting electrons in an atom from a core level to states near threshold.

EELS can be combined with transmission electron microscopy at fine spatial resolution. It usually has modest spectral resolution and is always rather damaging to the sample material. Many of the electrons scatter with small momentum transfer, causing chemical changes without contributing to the spectrum to be measured.

STXM with variable $x$-ray energy gives NEXAFS spectra at high spectral resolution and at a spatial resolution determined by the size of the zone plate x-ray focus. The radiation damage effects are typically two orders of magnitude lower than for EELS ${ }^{8}$ and the dose is under the explicit control of the experimenter.

Many organic materials are ideally suited for study by absorption spectroscopy, with very characteristic spectra. ${ }^{9,10}$ These same materials are also most sensitive to chemical changes induced by irradiation. The direct counting of transmitted photons behind a sample is the most efficient way to obtain an absorption spectrum, with the smallest number of incident photons. Radiation damage is still a concern. In a typical STXM study of organic material there may be features in the NEXAFS spectrum which are seen to change due to accumulated dose. If these most sensitive spectral features are identified the dose can be controlled and a measurement may be obtained twice from the same location on the sample before appreciable changes are seen.

\section{B. Spectra can be obtained from samples which contain water}

Many problems to which chemical imaging can be applied involve hydrated materials. For example, the study of samples from the environment with spatially concentrated contaminants should be made without removing the water. Such studies will also require the measurement of spectra at absorption edges other than carbon. By operating STXM at atmospheric pressure, usually in helium, we have convenient sample installation, and fewer restrictions on sample preparation. We exploit the controlled atmosphere to work at the oxygen and nitrogen edges, to study hydrated species, and to develop an instrument which can be used at absorption edges over a wide range of photon energy, from 150 to $900 \mathrm{eV}$.

Wet cells have been built in which hydrated precipitates and solutions can be examined. Spectra are obtainable through thin silicon nitride cell windows, even at the nitrogen $K$-edge.

\section{STXM can be polarization dependent}

Transitions contributing to features in NEXAFS spectra often involve electronic states which are not spherically symmetric, in systems which are partially aligned. In these situations the absorption depends on the polarization of the photons. Synchrotron light is naturally linearly polarized in the horizontal plane of the electron beam orbit of the storage ring. NEXAFS polarization dependence can be exploited to reveal the alignment of molecular orbitals. ${ }^{11,12}$

At beamline 7.0 a quarter wave plate phase retarder is installed which can convert the linearly polarized light to circular, with alternating helicity. ${ }^{13}$ This device uses multilayer films in transmission at energies around $100 \mathrm{eV}$ (too low to be practical in this STXM) and uses a magnetized iron film at the iron $L_{2}$ and $L_{3}$ edge (710 and $724 \mathrm{eV}$ ). This latter technique has made it possible to use magnetic circular dichroism contrast to observe magnetization patterns in thin iron samples.

\section{DESCRIPTION OF THE INSTRUMENT}

A pinhole is illuminated $3 \mathrm{~m}$ upstream from the STXM (this pinhole is common to both SPEM and STXM). The zone plate lens generates a demagnified image of the pinhole on the sample. An alternative illumination scheme may be employed in which the pinhole is removed and the beamline delivers approximately plane wave fronts to the zone plate lens. While the illumination is less certain in the latter case, the counting rate is higher and no degradation of the spatial resolution has been observed in practice.

Figure 2 shows the geometry of the vacuum window, the lens, the sample, the detector, and the order sorting aperture (OSA) which blocks the unfocused zero order light.

The zone plate focal length is proportional to the energy of the photons and the focusing is controlled by two stages, one on top of the other, which move the lens and vacuum window together over several millimeters, to cover the full range of photon energy. The parameters of the zone plates in use, and the technique for focusing and staying in focus during a NEXAFS scan, are discussed below.

Figure 3 shows the STXM mechanism, including the helium vessel which covers all the components from downstream and seals against the vertical support bulkhead. There are various motorized motions for alignment, prior to measuring a sample.

(1) The transverse $(x / y)$ position of the order sorting aperture must be checked and repositioned when the photon energy is changed by a large amount. Motorized stages encoded to $1 \mu \mathrm{m}$ accuracy are provided which also allow the OSA to be translated out of the system or positioned on the optical axis. 


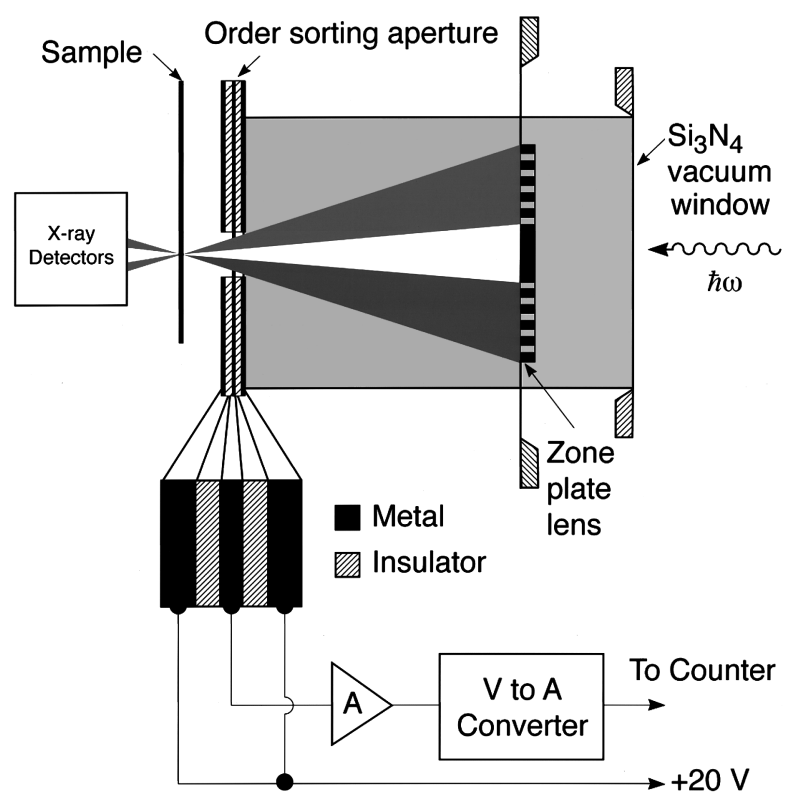

FIG. 2. Schematic arrangement of the windows, lens, order sorting aperture, the sample and the detector in STXM.

(2) The sample is held from a piezo scanning stage, which is itself carried on a pair of $x / y$ stages (Newport PM500) for coarse positioning and scanning. These coarse positioning stages can themselves be used to make an image but they cause a large mass to move so that they cannot make high resolution images except very slowly. In principle, they can be used with a step size as small as $50 \mathrm{~nm}$ but they are normally used to make large scale survey images at lower magnification. High resolution images are made over a $100 \mu \mathrm{m} \times 100 \mu \mathrm{m}$ area with the piezoelectric scanning stage (see below).

(3) The sample stage assembly can be moved along the optical axis $(z)$. This is important because of varying thicknesses of samples. The distance between the OSA and the sample surface should be as small as is safe (about $300 \mu \mathrm{m})$.

(4) Two detectors are installed (see below). They can be interchanged by horizontal transverse motion $(x)$ under

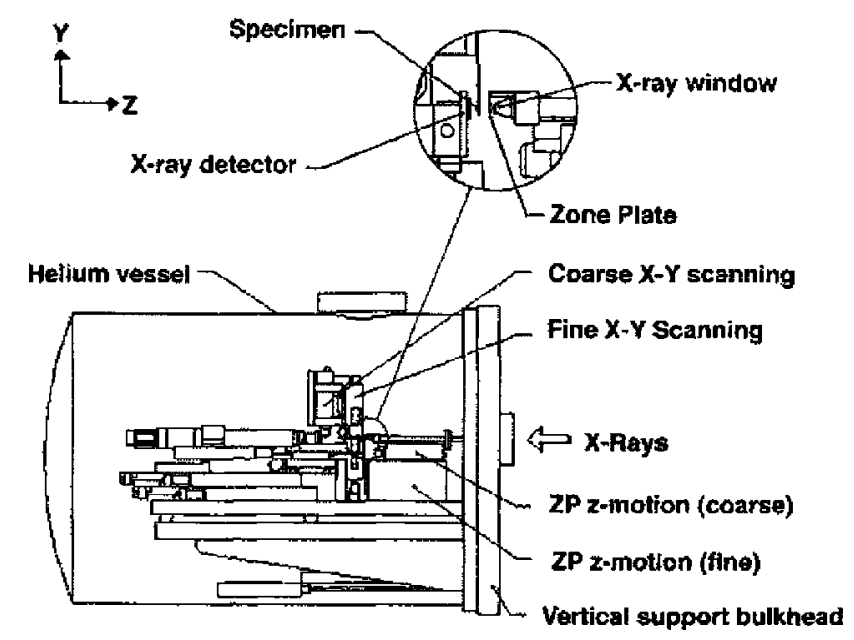

FIG. 3. Overall layout of STXM, showing the helium enclosure and the alignment stages. computer control and the $z$ position is also motorized, allowing them to be moved up close behind the sample.

All control and acquisition software is written using LABVIEW from National Instruments Inc. on a UNIX platform with motor control and data acquisition in VXI. We use National's MXI interface to the VXI crate. Images and spectra are written to a network disk, and are immediately accessible for processing using 'IDL' from Research Systems Inc. on another UNIX workstation adjacent to the experiment.

Two transmission detectors are currently installed.

(1) An analog silicon diode is used for alignment and for measurements at full intensity. It is from International Radiation Detectors Inc. and has an especially thin oxide layer on the front surface so that, except at photon energies below about $200 \mathrm{eV}$ and at the oxygen edge, the efficiency is effectively $100 \%$. Silicon requires an average of $3.6 \mathrm{eV}$ deposited per electron-hole pair. The detector has an active area about $5 \mathrm{~mm}$ in diameter, must be shielded from visible light, and has a dark current of $25 \mathrm{pA}$ at room temperature. Amplifier noise and ground loop fluctuations limit the use of this detector to situations with transmitted flux of more than about $10^{7}$ photons/s. The signal is amplified and acquired through a voltage to frequency converter which gives pulses to be counted.

(2) A pulse counting detector has recently been added and is now used almost all of the time. It consists of an efficient fast phosphor $\left(\mathrm{P} 43, \mathrm{GdO}_{2} \mathrm{~S}_{2}: \mathrm{Tb}\right)$ coated on a microscope cover slip. The phosphor is placed $1 \mathrm{~mm}$ downstream of the sample, and optically coupled to the lucite window of a hermetic enclosure which contains a photon-counting photomultiplier tube (PMT), R647P from Hamamatsu Inc. The phosphor converts each soft $\mathrm{x}$ ray to multiple visible light photons, some of which pass through the lucite window onto the photocathode of the PMT which is in-line downstream. The system shows an overall efficiency of $30 \%(90 \%)$ measured, respectively, at $300 \mathrm{eV}(650 \mathrm{eV})$ by comparison with the silicon photodiode (see Fig. 4). The commercial photon counting system (Hamamatsu) is linear up to rates of 15 $\mathrm{MHz}$ and easy to operate. We were led to use less efficient phosphors than the standard P31 zinc sulfide in order to avoid long lived fluorescence. Any visible light emitted more than $1 \mathrm{~ms}$ after the absorption would smear the images. The use of P43 was suggested by W. Meyer-Ilse.

Because of intensity fluctuations which arise in beamline 7.0 , it is advantageous to record a normalization signal along with the transmitted flux during spectral acquisition. The OSA is constructed to give such a signal. A photocurrent is measured from an active area of metal intercepting all of the light passing through the window of the zone plate support membrane, except the first order light passed by the $35 \mu \mathrm{m}$ OSA pinhole. The surrounding metal supports of the OSA are biased positive to draw off these photoelectrons (see Fig. 2). This detection scheme also responds to ionization in the microscope atmosphere, which depends on its composition. The OSA signal gives a valid normalization except at the oxygen and nitrogen edges. It is typically a few picoamps of current, which is amplified and passed through a V-F converter to be counted as pulses along with the transmitted flux. 

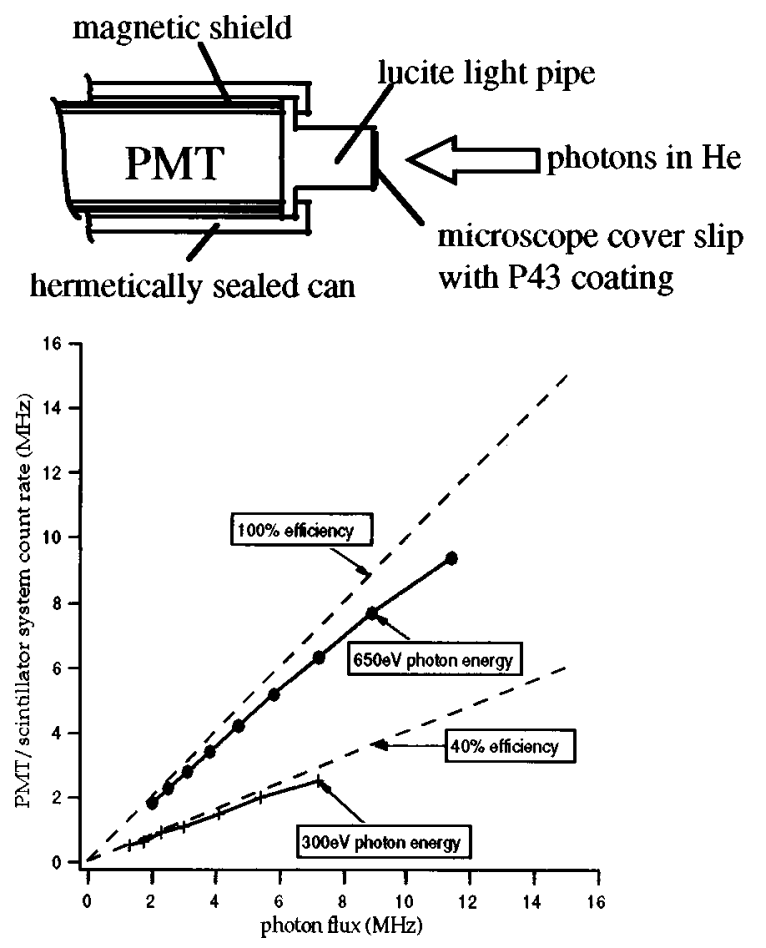

FIG. 4. Measured efficiency and linearity of the phosphor-photomultiplier detector system.

Samples are mounted on an aluminum frame which can carry eight $3 \mathrm{~mm}$ diam transmission electron microscope (TEM) grids. This holder clips to a kinematic mount on the moving platen of the piezo scan stage $5 \mathrm{~cm}$ above the beam (see Fig. 5). The sample holder is reproducibly positioned to within about $20 \mu \mathrm{m}$ so that samples can be removed and replaced allowing different experiments to switch in and out. Samples are photographed using a visible light microscope before they are loaded into STXM so that the coarse X-ray images can be navigated to the fine features of interest.

The scan stage is a monolithic $x / y$ flexure from Queensgate Instruments Inc., driven by piezo-electric transducers with integral capacitance encoders. It has a moving platen nominally traversing $100 \mu \mathrm{m}$ in $x$ and $y$, although we find the range reduced somewhat to about $80 \mu \mathrm{m}$ in practice. We acquire images of (typically) $100 \times 100$ pixels with a counting time of $1 \mathrm{~ms}$ per pixel. The stage is driven stepwise, always in the same direction, and the small-step settle time of the capacitance encoder feedback loop is about $2 \mathrm{~ms}$. The stage is intended to be used about the center of its moving platen, but we have raised it $5 \mathrm{~cm}$ and our sample is held below. This introduces Abbe errors whereby the slight rotation of the platen converts to unwanted transverse motion at the end of the vertical lever arm, and gives a few percent image distortion.

The most difficult mechanism in the instrument is the $z$-stage which moves the zone plate along the optical axis to retain the focus during a NEXAFS spectral scan. In measuring the absorption spectrum through the carbon edge from 280 to $320 \mathrm{eV}$, say, the focal length of our $80 \mathrm{~nm}$ zone plate (see below) changes from 3.62 to $4.14 \mathrm{~mm}$. The lens must translate $0.52 \mathrm{~mm}$ to remain in focus. It is necessary that this motion take place precisely along the optical axis, a straight

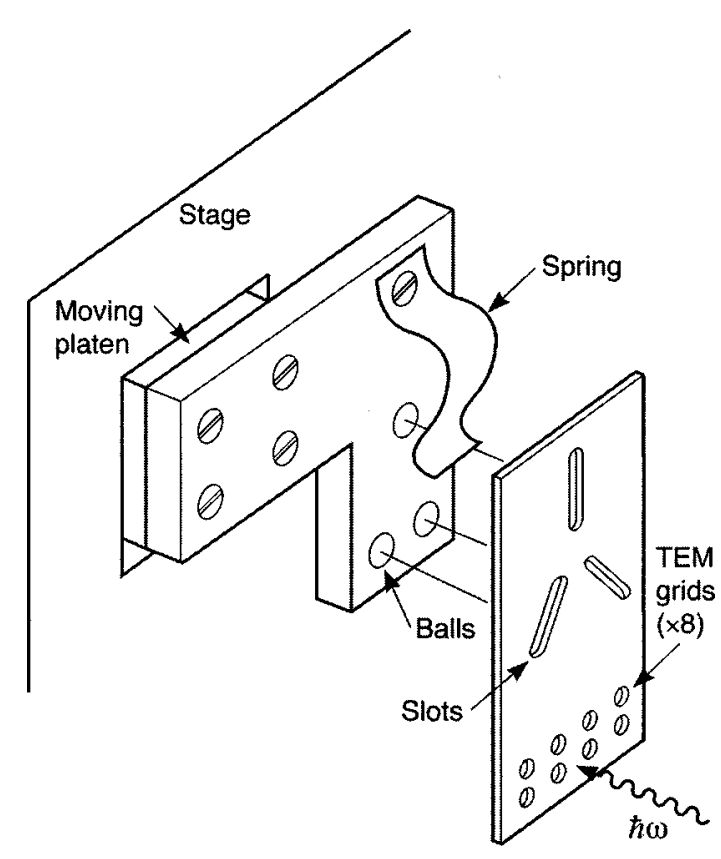

FIG. 5. Samples are mounted on a frame held to the moving platen of the scan stage in a kinematic mount. Samples can be removed and replaced with a reproducibility of about $20 \mu \mathrm{m}$.

line joining the center of the zone plate with the center of the pinhole $3 \mathrm{~m}$ upstream. Any error represents transverse walk of the spot on the sample and smears the area over which the spectrum is measured. The sample must be uniform over this area, otherwise spectral artifacts will appear. The transverse walk must be minimized if small features are to be analyzed.

We have built a flexure stage to move $0.8 \mathrm{~mm}$ along the optical axis using deformed sheet metal diaphragms, as shown in Fig. 6. More conventional flexure designs to cover this large range would be too large and bearing stages are not sufficiently reproducible. Our flexure does not move in a completely straight line. Figure 6 shows measurements of the unwanted transverse motion (run-out) measured by interferometry before installation. We observed about $0.2 \mu \mathrm{m}$ runout, with hysteresis and directional dependence, but we have found the run-out to be reproducible beyond the precision of the interferometer measurements to the limit of our x-ray measurements. There is a software "compensation" algorithm which moves the $x / y$ scanning stage according to the measured misalignment and measured run-out to keep the spot at the same location on the sample and eliminate the effects in the data. Performance is discussed below.

The zone plates currently in use are from a batch made by Erik Anderson at IBM, Yorktown Heights, in 1995. They are intended as amplitude zone plates in this range of photon energy, with opaque zones. They are used in two sizes: (a) $200 \mu \mathrm{m}$ in diameter, $80 \mathrm{~nm}$ outer zone width with a $60 \mu \mathrm{m}$ diameter central stop and (b) $90 \mu \mathrm{m}$ in diameter, $45 \mathrm{~nm}$ outer zone width with a $40 \mu \mathrm{m}$ diam central stop. The zones are $100 \mathrm{~nm}$ thick, gold on a $100 \mathrm{~nm}$ silicon nitride membrane. The first order focal length is given by

$$
f=D \delta r_{N} / \lambda
$$

where $D$ is the diameter, $\delta r_{N}$ is the outer zone width, and $\lambda$ 

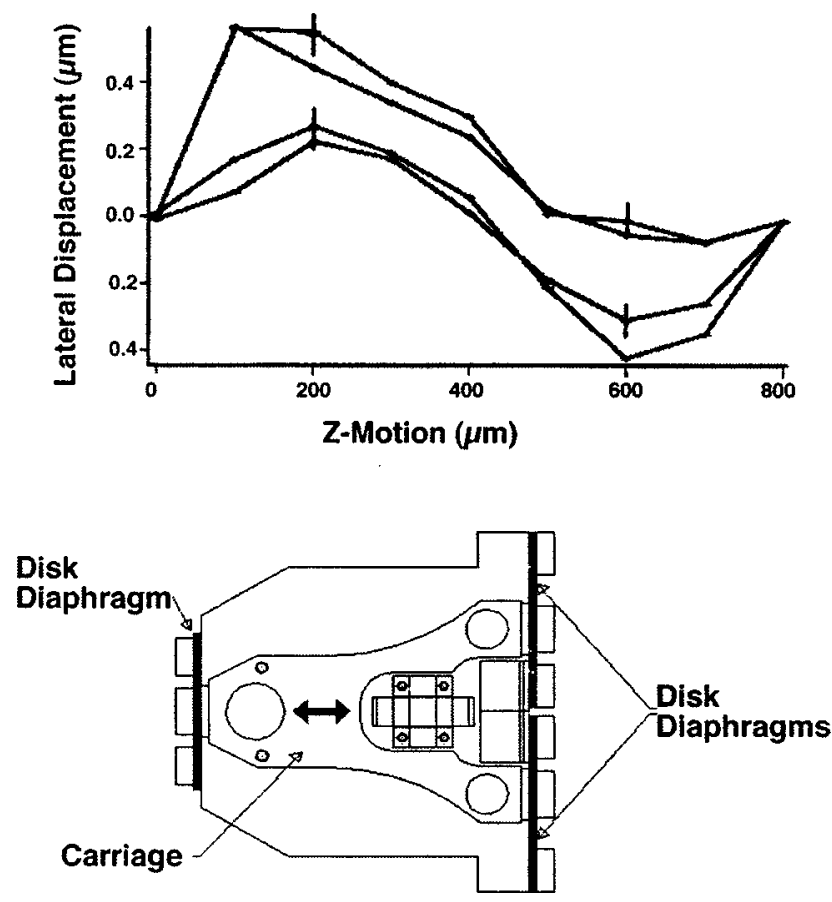

FIG. 6. Design and performance of the NEXAFS $z$ flexure which moves the zone plate lens longitudinally to stay in focus. The measured run-out is shown, including directional dependence (hysteresis). Errors are the estimated uncertainty of the interferometric measurements.

is the photon wavelength. The focal length has a value of: (a) $3.9 \mathrm{~mm}$, (b) $0.9 \mathrm{~mm}$, respectively, at the carbon edge.

Windows are silicon nitride grown by chemical vapor deposition on a polished silicon wafer which is then etched away with $\mathrm{KOH}$. These windows are fabricated by Dino Ciarlo at Lawrence Livermore National Laboratory. The vacuum window on the beamline is $160 \mathrm{~nm}$ thick, $250 \mu \mathrm{m}$ square, and has never broken. The zone plate lens carrier membrane is about $100 \mathrm{~nm}$ thick, and $300 \mu \mathrm{m}$ square. Figure 7 shows the transmission of $100 \mathrm{~nm}$ silicon nitride across the interesting range of photon energies.

A helium atmosphere is established in the microscope vessel once the sample holder is installed and the beam introduced. Normally we purge the air by introducing helium into the top of the tank and venting from the bottom. This causes the signal to rise about a factor of 20 , depending on

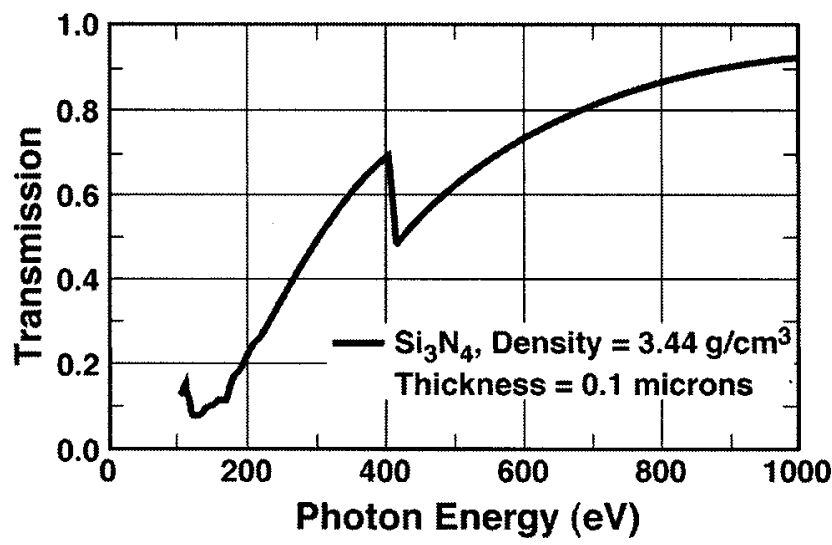

FIG. 7. Soft $\mathrm{x}$-ray transmission of $100 \mathrm{~nm}$ silicon nitride, across the energy range of interest. (Courtesy E. Gullikson) the photon energy and the corresponding working distance. For spectral measurements at the nitrogen or oxygen edge, purging will not produce a sufficiently pure helium environment and fluctuations of the residual oxygen or nitrogen content are problematic. In these cases the vessel is pumped, to $250 \mathrm{~m}$ Torr, which is as low as it will go with all the motors inside. A subsequent helium fill is sufficiently pure, so that there is little atmospheric absorption signal in the measured spectra and normalization is reliable. High voltage feedthroughs in the bulkhead have been adapted to prevent breakdown in helium. The scanning stage high voltage (170 $\mathrm{V})$ poses no problem. The photomultiplier tube is hermetically sealed against the helium and held in air at atmospheric pressure. Cable connections carry the bias $(1100 \mathrm{~V})$ through helium without breakdown.

\section{OPERATIONAL PROCEDURES}

Although the brightness of beamline 7.0 is a powerful advantage, there are practical difficulties. Sharing the beamline means that time is spent configuring the optics and steering the beam back into the microscope before use. During microscope development the beamline has been shared by literally hundreds of visiting scientists, which has complicated the task.

Another challenge is that the high brightness source does not overfill the apertures by the large factors seen in implementations of diffraction limited systems on older light sources. This leads to larger variations in signal due to motion of the photon beam.

Here is a description of the operations necessary for spectromicroscopy data acquisition:

(1) For work at a certain edge, the correct monochromator grating is selected, the photon energy is selected in the middle of the NEXAFS range, and the refocus mirrors are adjusted to put the beam into the microscope.

(2) With the OSA removed from the beam path, the mirrors are steered to maximize the flux passing through the zone plate, into one of the transmission detectors. This establishes the line which the beam naturally takes.

(3) The pinhole can be placed in the beam 3 m upstream and its transverse position is optimized for maximum transmission. The nominal pinhole size (e.g., $60 \mu \mathrm{m}$ diameter) is that which will be demagnified to a spot on the sample of size equal to the diffraction limit of the zone plate lens. We often use an undersize pinhole (e.g., $25 \mu \mathrm{m}$ diameter) to cut down the intensity to control radiation damage.

(4) The OSA is used as a scanned pinhole (35 $\mu \mathrm{m}$ diameter) to measure the illumination uniformity of the zone plate on its window, and to adjust the beam steering if necessary. Then the OSA is placed at the center of the measured pattern, which shows the first order light coming to a focus. The OSA must be accurately positioned $(+/-2 \mu \mathrm{m}$ for the $45 \mathrm{~nm}$ zone plate) to eliminate transmission of unfocused zero order light, which would give blurred ghosts in the image.

(5) Now we are ready to focus on the sample. A STXM image with a contrast feature is required (this may be the edge of a grid bar). An $x / y$ line scan is defined across an 
intensity variation in the image. The software focus algorithm performs repeated $x / y$ scans along this line with varying focus conditions (zone plate $z$ positions) and one sees the position at which the focus is sharpest. The depth of focus is typically a few $\mu \mathrm{m}$ so some care is required. This focusing is made using the PM500 zone plate $z$ stage atop the $z$ flexure with the flexure at mid-range. The use of the flexure is reserved for NEXAFS spectral scans.

(6) With a focused STXM image on the computer screen, made using the piezo $x / y$ scan stage, one can navigate to areas of interest. The photon energy must be chosen correctly to render features visible with chemical contrast. With the photon spot stationary on the sample surface, an absorption spectrum can be acquired. Undulator moves are usually made at each energy step and a shutter is provided to automatically cut off the beam during undulator and diffraction grating motion ( $200 \mathrm{~ms}$ per move), to protect against unnecessary radiation damage. An adjustable dead-band is provided in the undulator control algorithm which can be increased to allow several energy points to be measured before the gap is changed, or the undulator motion can be disabled. The shutter is also used to record the detector dark counts before and after the spectral scan. A counting period of $200 \mathrm{~ms}$ per energy point is typical. Normalization signals are recorded along with the count of transmitted photons, then the sample is removed, and the measurement repeated. If $t_{i}, n_{i}$ are the transmitted signal and the normalization signal (dark levels subtracted) with the sample in, and $t_{o}, n_{o}$ are the repeated measurement with the sample out, then the absorption cross section is proportional to:

$$
\ln \left[\left(t_{o} \cdot n_{i}\right) /\left(t_{i} \cdot n_{o}\right)\right]
$$

(7) The thickness of the sample should be such that there is at least about one absorption length of the species of interest. Samples which are too thin or too thick give noisy spectra, either because there is too little reduction in count rate at the edge, or too few counts after transmission. Samples can be several absorption lengths thick before the latter condition is encountered. If the species of interest is dilute, the thickness can be increased, and atoms present at the level of about $2 \%$ can be studied. The sample must be uniform across the spot size of the focused photon beam, otherwise the spectrum is distorted. Some higher order light is delivered by the beamline at integer multiples of the desired photon energy and is present at the STXM focus at the level of about $1 \%$. This can distort the spectra obtained from thick samples unless the amount of higher order light is determined and subtracted from the signals before the ratio is made.

(8) The tuning of the compensation algorithm is checked prior to the measurement of submicron features. The algorithm moves the $x / y$ position of the sample to eliminate the effects of transverse stage run-out as the zone plate is moved in $z$ by means of the flexure stage to remain in focus through the NEXAFS scan. Features in the image which retain contrast are imaged at various energies through the NEXAFS range with the compensation active, to ensure there are no
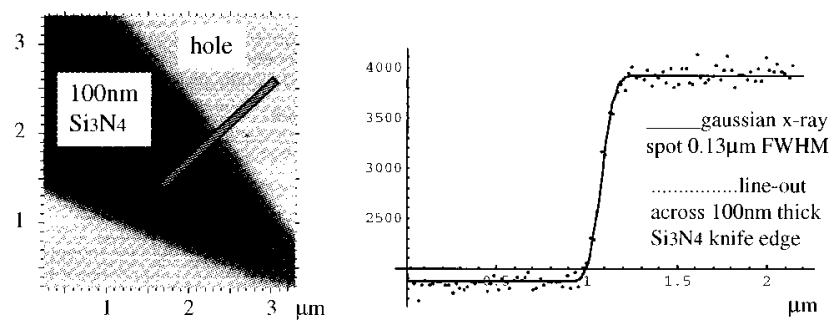

FIG. 8. Spatial resolution measurements using the edge of a thin fragment of a silicon nitride window. The curve superimposed on the measured edge profile is computed as a Gaussian convoluted with an edge. The vertical scale is the number of photons counted in transmission. Noise is somewhat worse than statistical, showing the magnitude of beam motion (under favorable conditions) which causes the illumination intensity to vary.

problematic shifts in the image position. If such shifts are seen the algorithm parameters can be adjusted.

\section{EARLY PERFORMANCE}

We have measured $1.7 \times 10^{8}$ photons/s transmitted through the helium atmosphere with a spectral width $1 / 3000$ in the zone plate first order focus spot at $320 \mathrm{eV}$, with the storage ring running at $1.9 \mathrm{GeV}, 400 \mathrm{~mA}$. Images can be made with a counting time on the order of $1 \mathrm{~ms}$ per pixel. At higher photon energies the intensity increases, as the windows become more transparent, until the beamline output decreases above $500 \mathrm{eV}$. In order to maintain uniform overfilling of the microscope pinhole and the zone plate lens it is necessary to maintain optimized tuning of the undulator gap, to produce the required photon energy in the central cone. Optimized beam steering must be maintained for the same reasons. Turning the intensity down for radiation sensitive measurements is best accomplished by reducing the monochromator exit slit width, typically to $10 \mu \mathrm{m}$, reducing the diameter of the pinhole, typically to $25 \mu \mathrm{m}$, and by choosing a less powerful undulator harmonic.

Figure 8 shows an image and a profile of a fragmented silicon nitride window made using the $45 \mathrm{~nm}$ zone plate, demagnifying a $25 \mu \mathrm{m}$ pinhole by a factor of 1000 . The broken remnants of these windows form sharp points and well defined edges, good for $\mathrm{x}$-ray spot position and size measurements. The effective size of the spot is the most relevant spatial resolution parameter. We discuss the measured full width half maximum (FWHM) of the x-ray spot, and find it larger than the diffraction limit. Several effects contribute to its size and we assume a Gaussian profile for simple analysis. A Gaussian is convoluted with a step function to represent a line across the image of the edge. The resulting curve is fit to the data with $0.13 \mu \mathrm{m}$ FWHM. Normally one considers a diffraction limited zone plate spot to have a diameter $1.2 \delta r_{N}(=0.055 \mu \mathrm{m}$ in this case). A diffraction limited spot produced by this zone plate (with central stop) would have a FWHM of $(0.4 \lambda)$ /numerical aperture (NA) $=0.040 \mu \mathrm{m},{ }^{14}$ with extended fringes.

There are several other possible contributions to the size of the x-ray spot.

First, the geometrically demagnified pinhole is a circle diameter $0.025 \mu \mathrm{m}$. 
Second, in order to maintain alignment this STXM is supported without vibration isolating air pads. Ambient vibrations of the scanning stage and zone plate are measured with accelerometers and exhibit several peaks at acoustic frequencies with amplitudes on the order of $0.010 \mu \mathrm{m}$. This indicates that we are close to the point where ambient vibrations limit the spatial resolution. We sometimes observe streaking of small features when measured quickly with $\mathrm{x}$ rays, consistent with horizontal and vertical vibrations at frequencies from about 10 to $50 \mathrm{~Hz}$. These are at the $0.050 \mu \mathrm{m}$ level. These effects may be due to vibrations or to electrical noise in the scan control. They are under investigation. fect.

Finally, it is also possible that the zone plate is imper-

For spectromicroscopy, the spectrum is as important as the image. The goal is to measure absorption spectra from small features and the minimum size feature that can be measured is a crucial parameter.

In the first mode of spectral acquisition we want to extract absorption spectra by illuminating a localized spot on the sample. Clearly the x-ray spot size is a limit, but in STXM the possibility of transverse motion of the spot on the sample as the lens moves longitudinally (to stay in focus through the NEXAFS scan) may limit the technique further. Our software procedure, which moves the sample in $x / y$ to compensate for this effect, is successful up to the level that the effect is measured, and is reproducible at this level from scan to scan. Figure 9 shows the results. Here we see a series of images of submicron polymer precipitate particles measured at energies through the oxygen $K$-edge NEXAFS range, taken with the compensation algorithm operational. The apparent shift of the images through this typical carbon NEXAFS range has been reduced to less than $0.15 \mu \mathrm{m}$ so that spectra can be acquired from uniform regions of the image as small as about $0.2 \mu \mathrm{m}$ without artifacts due to transverse walk of the spot.

It is necessary to accurately reposition the x-ray spot on sample features seen in a previously scanned image. One concern is thermal drift, since some time may elapse between image acquisition and navigation for spectroscopy. With the instrument enclosure sealed, we observe tenths of micron thermal drift between the sample and the lens on a timescale of tens of minutes. These effects are detected by stepping transversely across image features at fixed energy prior to dwelling for a spectral measurement. Figure 9 shows a successfully navigated image. Radiation damage is seen at the point of dwell on the shell of a polymer precipitate particle after a number of spectral measurements.

At the time of writing the effective spatial resolution for spectromicroscopy in this first mode of spectral acquisition is set by (a) the effective spot size, (b) the precision of the run-out compensation (which is based on data extracted from x-ray images), and (c) thermal drift. All three effects have similar magnitude.

In the second mode of spectral acquisition "stacks" of images are acquired over a suitable range of photon energies. For example, 100 images are acquired at unequally spaced values of the photon energy through the NEXAFS range. This mode takes advantage of the high count rates and mini-
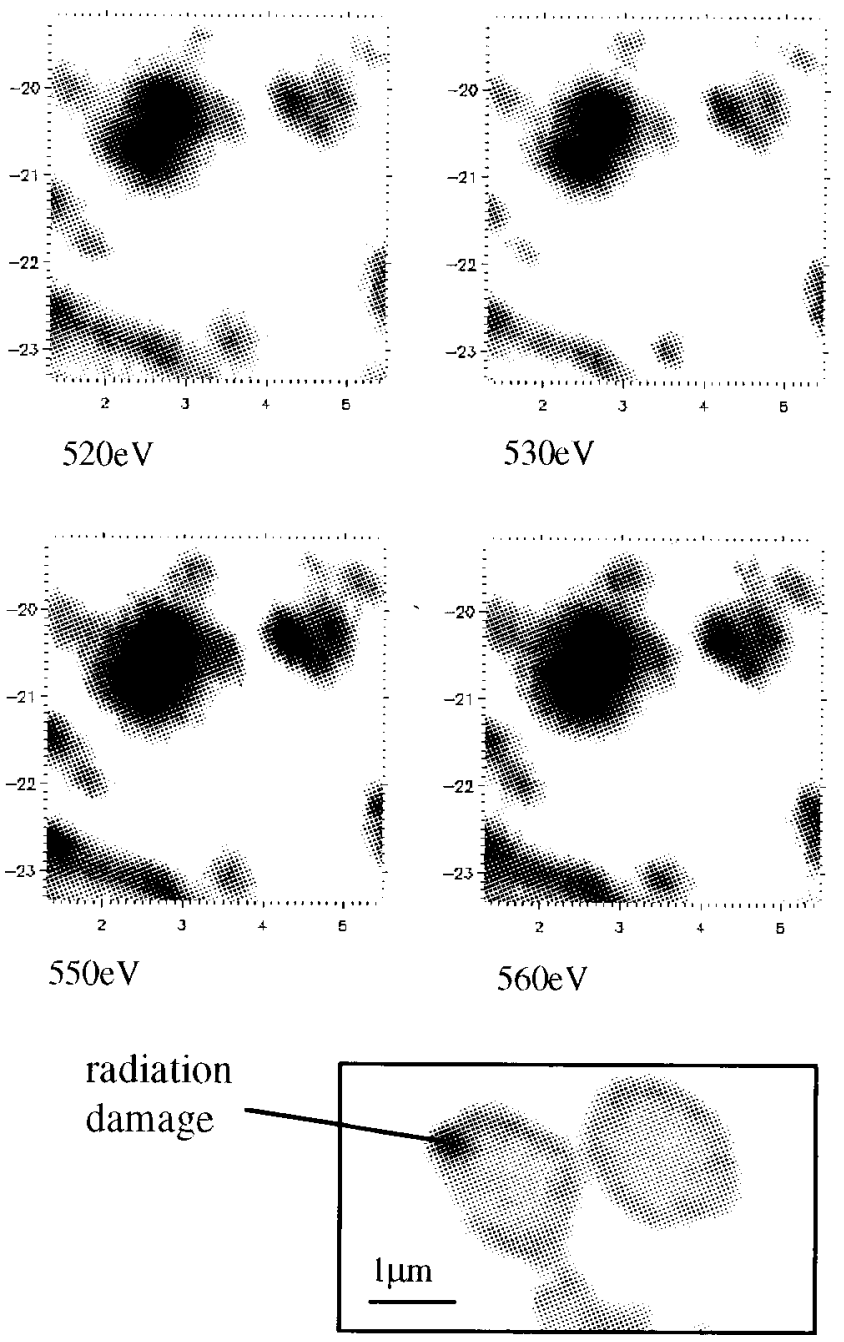

FIG. 9. Images of submicron polymer precipitates transversely compensated during acquisition, as the photon energy is stepped through the oxygen $1 \mathrm{~s}$ NEXAFS range. The residual motion of the images in the frame is less than $0.15 \mu \mathrm{m}$. Also shown is an image made following accurately placed spectroscopic measurements which resulted in radiation damage to the measured shell of the precipitate particle.

mizes the overhead due to undulator gap changes. These sequential images are shifted in $x$ and $y$ as necessary to register with one another to avoid the effects of run-out and thermal drift. Spectra are obtained by summing pixels over selected image features. These analyses are made using computer programs provided by C. Jacobsen. ${ }^{15}$ If an area without spectral features is included in the images, normalization is built into the data set. Such developments are also underway at NSLS. The data acquisition time will be considerably reduced at the ALS.

\section{EARLY RESULTS}

Figure 10 shows sectioned Kevlar fibers imaged at the C, $\mathrm{O}$, and $\mathrm{N} K$-edges by absorption of polarized x-rays with $E$ vector horizontal. The polymer chains point along the fiber and the aromatic rings and carbonyls are oriented preferentially in the radial direction. This means that $\pi^{*}$ orbitals into which $\mathrm{C}, \mathrm{O}$, and $\mathrm{N} 1 s$ electrons can be excited are all preferentially tangential. This orientation is reflected in the co- 


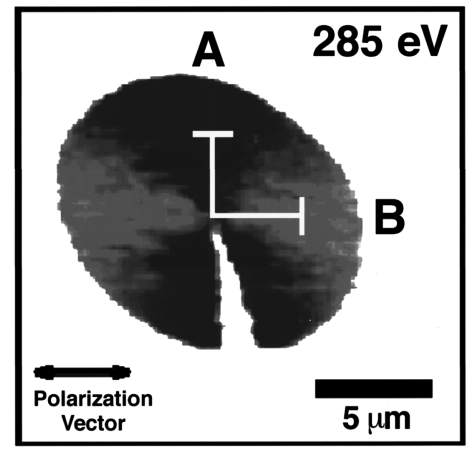

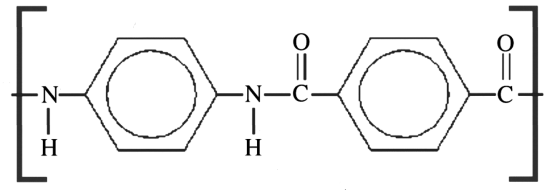

Kevlar
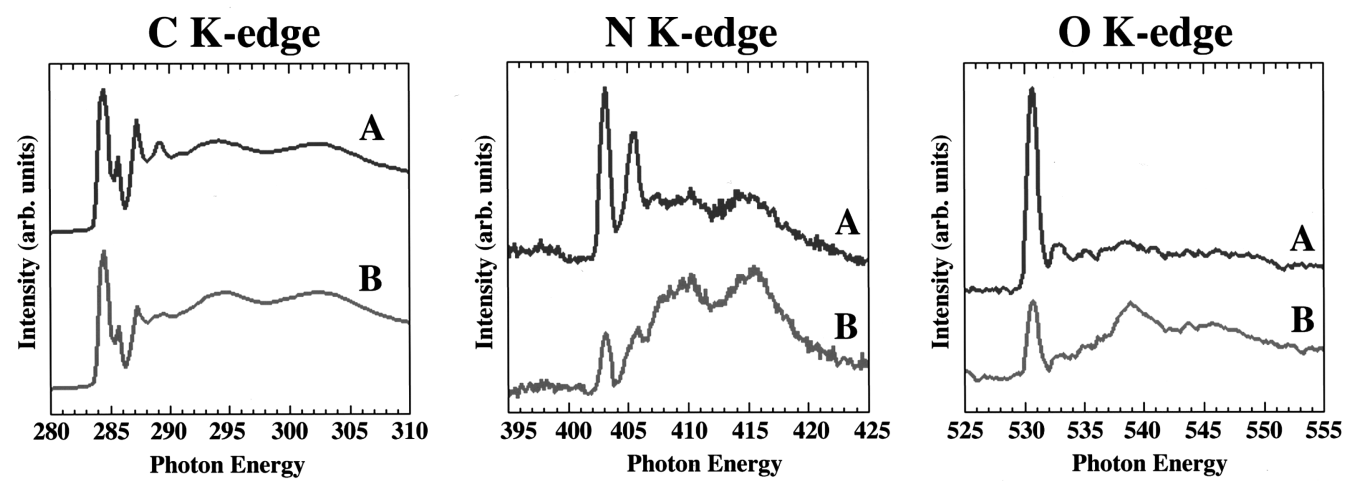

FIG. 10. Sectioned Kevlar fibers have been imaged to demonstrate the polarization sensitivity of STXM. The photon $E$ vector is horizontal. The $\pi^{*}$ orbitals are oriented preferentially radially and this is reflected in the changing ratio of $\pi$ to $\sigma$ absorption, especially in the case of nitrogen and oxygen, which occupy unique sites.

sine squared dependence of the absorption cross section at the energy of the transitions into $\pi$ orbitals. The opposite is true for excitation into $\sigma$ orbitals. The degree of orientation can be quantified, ${ }^{12}$ at least for the $\mathrm{O}$ and $\mathrm{N}$ atoms, which occupy unique sites in the molecule.

Figure 11 shows images and localized C $K$-edge NEXAFS spectra of a organic-rich soil sample (pine ultisol, $\mathrm{Pu}-$ erto Rico) in its original form (with water present). The wet sample was mounted between two silicon nitride windows (160 nm thick). Large density variations, silicate, and ironoxide particles, and filigree organic structures are visible in
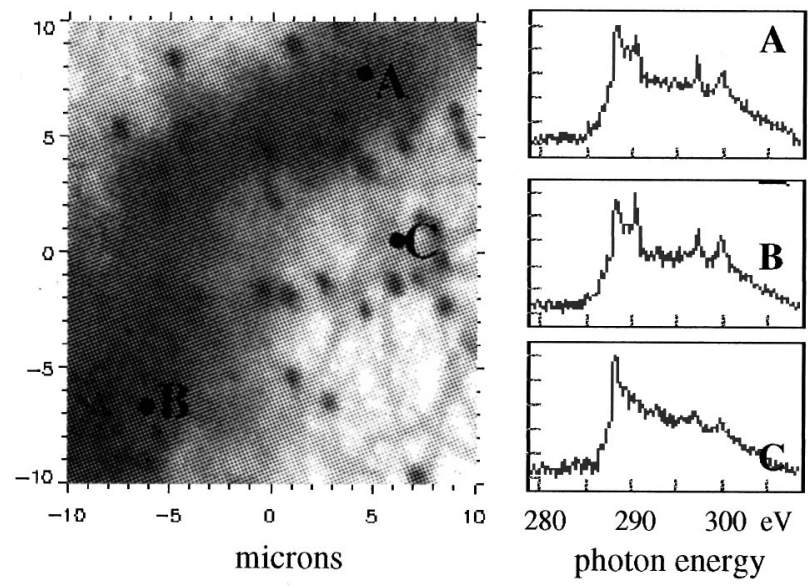

FIG. 11. Wet soil samples have been studied for their contamination chemistry. In this example the peaks around $290 \mathrm{eV}$ are $p$ transitions in specific organic functional groups, the two peaks around $300 \mathrm{eV}$ are $L$-edge absorption in potassium. The spatial location of the potassium is associated with the presence of specific organic groups. the STXM image. These in situ measurements involve an original soil sample not exposed to any kind of chemical treatment, with uncertain thickness and several orders of magnitude attenuation of the transmitted flux. The NEXAFS spectra show characteristic $1 s-\pi^{*}$ peaks from organic functional groups. The spectra clearly exhibit different groups (corresponding to $1 s-\pi^{*}$ transitions at different energies). Work is underway to obtain NEXAFS spectra from chemically extracted soil organic molecules (humic and fulvic acids), against which these microscopic results will be compared. ${ }^{16}$

Figure 12 is an image revealing magnetic domains in a $26 \mathrm{~nm}$ thick, demagnetized Fe film. This image was produced using magnetic circular dichroism (MCD) for absorption contrast. Transmission through a saturated magnetic $\mathrm{Fe}$ film provides circularly polarized light from the linearly polarized undulator beam by preferentially absorbing one helicity with respect to the other. This resonant circular polarizer ${ }^{13}$ is placed upstream of the STXM so that the beam focused onto the sample has some degree of circular polarization. Since Fe (and most) thin magnetic films have magnetization $\mathbf{M}$ in-plane, both polarizer and sample need to be positioned away from normal incidence so that the scalar product of the wave vector $\mathbf{k}$ and $\mathbf{M}$ is nonzero. In this case the sample normal is rotated $35^{\circ}$ away from $\mathbf{k}$ and the sample oriented about its normal to maximize k.M. Domains in the demagnetized sample are observed as the orientation of $\mathbf{M}$ changes by $180^{\circ}$ across domain walls. The contrast can be reversed simply by reversing the magnetization in the upstream film either by rotation or electromagnetically. Quantitative studies of the variation of magnetization with posi- 

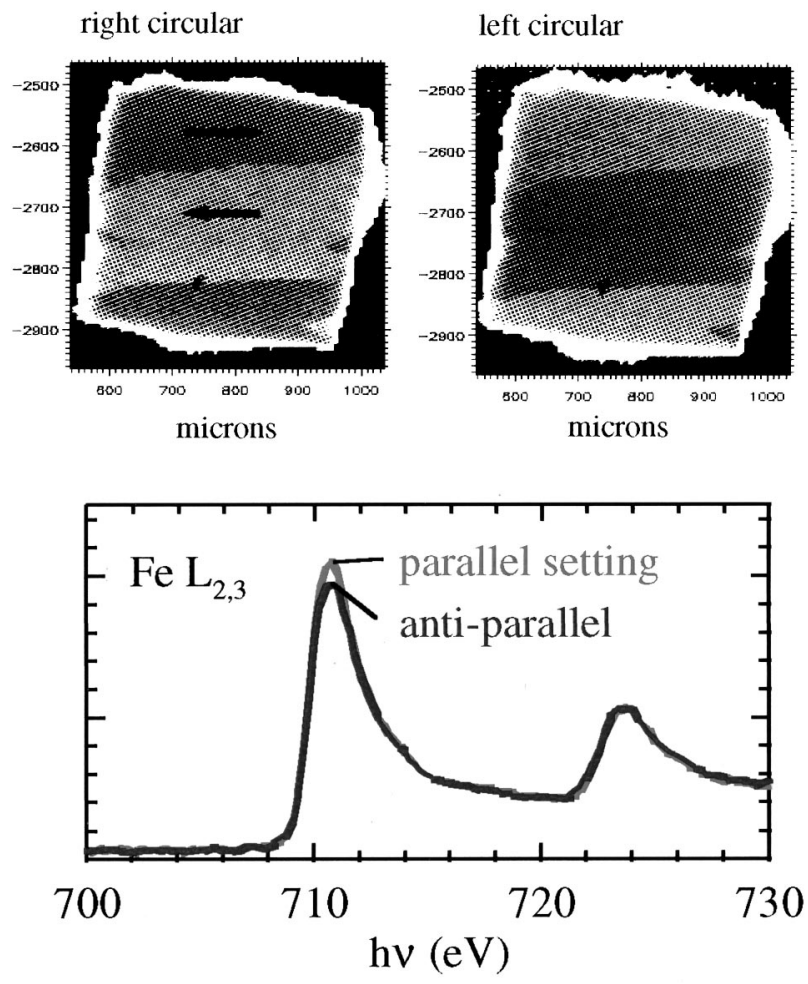

FIG. 12. Magnetic contrast has been achieved using transmission Fe films as resonant circular polarizers in the incident beam at the $L 3$ white line (708 $\mathrm{eV}$ ) where magnetic circular dichroism is maximum. The polarizer can be flipped parallel or anti-parallel to the longitudinal magnetization vector of the sample. Domains are observed in demagnetized Fe films mounted in STXM with normal at $35^{\circ}$ to the incident photons.

tion are possible using this technique, as are studies of the magnetization distributions in different magnetic layers in magnetic multilayer structures.

Another area of application of the STXM is in the study of the chemical processes taking place within and on the surfaces of microcrystalline components of soils, including both mineral and clay particles. The chemically active fraction of clays, which are alumino-silicate layered crystals, are the microscopic particles near $1 \mu \mathrm{m}$ in size. In order to study these microcrystals in their natural state, it is necessary to retain a layer of liquid water, since the clays naturally absorb water which significantly changes their physical volume and chemical activity. The STXM allows us to probe the changes in chemical composition within individual clay particles in the natural, fully hydrated state. Figure 13 shows a clay par-
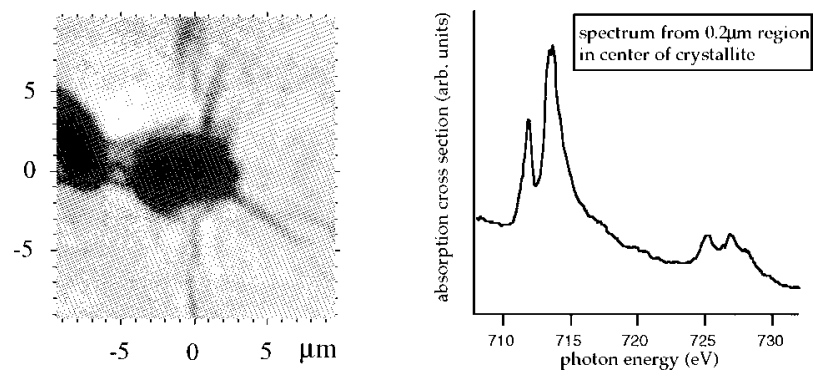

FIG. 13. Fe in solution absorbed by small clay particles. The x-ray image of the wet sample locates the particle, local concentration of contaminant is high in the center and the Fe $L$-edge spectrum reveals the ionic state. ticle, which is intercalated with $\mathrm{Fe}$ ions and fully hydrated while imaging. The concentration of $\mathrm{Fe}$ is high enough to perform in situ near-edge spectroscopy on a small region of a single clay particle, held between the windows of a "wet cell." The $L$ edge spectrum of the Fe can be used to determine the $\mathrm{Fe}$ (II,III) valence distribution. Comparison to spectra of known Fe salts in solution (also measured with this instrument ${ }^{17}$ ) shows that this particular sample is principally $\mathrm{Fe}(\mathrm{III}) .{ }^{18}$ Local concentrations of dopant species should be on the order of $1 \%$ or higher for this measurement technique.

\section{DISCUSSIONS}

We have extended the STXM technique to higher photon energies and faster data rates in an instrument at an undulator beamline at the Advanced Light Source.

We have developed a capability of measuring local absorption spectra at the oxygen and nitrogen $K$-edges at atmospheric pressure, in addition to the very important carbon $K$-edge. These are the three most important constituents of man-made organic materials, and this capability is an excellent match to the needs of $\mathrm{x}$-ray polymer spectromicroscopic analysis.

The STXM is capable of resolving the chemical state of transition metal species in solution at $0.2 \mu \mathrm{m}$ resolution. It is ideally suited to studies of the microbe-mineral interface, since it is equally capable at the lower energy $\mathrm{C}, \mathrm{N}$, and $\mathrm{O}$ edges and at the previously inaccessible $L$-edges of the transition metals. We have developed a capability for measuring wet thick samples from the environment, studying the spatial distributions of specific organic functional groups, and analyzing dopant species at local concentrations on the order of $1 \%$.

Magnetic domains in the bulk of magnetic thin films can be imaged using circular polarized photons at the $\mathrm{Fe}, \mathrm{Ni}$, and Co $L$-edges. This allows layer-specific studies of the domain structure of magnetic mutilayers.

Our count rates are about ten times higher than in implementations elsewhere so that data rates for image sequences are greatly improved.

We have not quite achieved diffraction limited spatial resolution. The development of the instrument proceeds.

User programs are now underway in the areas of polymer science, environmental chemistry, and magnetic thin films.

\section{ACKNOWLEDGMENT}

This work was supported by the Director, Office of Energy Research, Office of Basic Energy Sciences, Materials Sciences Division of the U.S. Department of Energy, under Contract No. DE-AC03-76SF00098

${ }^{1}$ J. Kirz, H. Ade, R. E. Botto, G. D. Cody, J. Fu, C. Jacobsen, S. Lindaas, W. F. Mangel, W. J. McGrath, V. Oehler, J. Van t'Hof, S. Williams, S. Wirick, and X. Zhang X-Ray Microscopy IV, Proceedings 4th International Conference (Inst. Microelectr. Tech., Chernogolovka, Russia, 1994).

${ }^{2}$ G. R. Morrison, P. A. F. Anastasi, M. T. Browne, C. J. Buckley, R. E. Burge, P. S. Charalambous, G. F. Foster, A. G. Michette, D. Morris, J. R. Palmer, G. E. Slark, P. M. Bennett, and P. J. Duke, X-Ray Microscopy III, Springer Series in Optical Sciences (Springer, Berlin, 1992), Vol. 67, p. 139. 
${ }^{3}$ J. Kirz, C. Jacobsen, and M. Howells Q. Rev. Biophys. 28, 33 (1995).

${ }^{4}$ C. Jacobsen, S. Williams, E. Anderson, M. T. Browne, C. J. Buckley, D.

Kern, J. Kirz, M. Rivers, and X. Zhang, Opt. Commun. 86, 351 (1991).

${ }^{5}$ H. Ade, X. Zhang, S. Cameron, C. Costello, J. Kirz, and S. Williams, Science 258, 972 (1992).

${ }^{6}$ J. Stohr, NEXAFS Spectroscopy (Springer, Berlin, 1992).

${ }^{7}$ J. Kirz, J. Opt. Soc. Am. 64, 301 (1973).

${ }^{8}$ E. G. Rightor, A. P. Hitchcock, H. Ade, R. D. Leapman, S. G. Urquhart, A. P. Smith, G. Mitchell, D. Fischer, H. J. Shin, and T. Warwick, J. Phys. Chem. B B101, 1950 (1997).

${ }^{9}$ V. Carravetta, H. Agren, L. Petterson, and O. Vahtras, J. Chem. Phys. 102, 5589 (1995).

${ }^{10}$ S. Urquhart, A. P. Hitchcock, E. Rightor, and R. Priester, J. Polym. Sci., Part B: Polym. Phys. 33, 1603 (1995).
${ }^{11}$ H. Ade and B. Hsiao, Science 262, 1427 (1993).

${ }^{12}$ A. P. Smith and H. Ade, Appl. Phys. Lett. 69, 3833 (1996).

${ }^{13}$ J. Kortright, S. K. Kim, T. Warwick, and N. V. Smith, Appl. Phys. Lett. 71, 1446 (1997).

${ }^{14}$ L. Jochum and W. Meyer-Ilse, Appl. Opt. 34, 22 (1995).

${ }^{15} \mathrm{C}$. Jacobsen (private communication).

${ }^{16}$ S. C. B. Myneni, J. T. Brown, W. Meyer-Ilse, G. A. Martinez, A. Garcia, and A. Warwick, Abstracts of the AGU National Meeting, San Francisco, 1997.

${ }^{17} \mathrm{~J}$. Rothe (private communication).

${ }^{18}$ T. Grundl, S. Cerasari, A. Garcia, T. Warwick, H. Ade, and B. P. Tonner, Compendium of User Abstracts and Technical Reports 1993-1996, LBNL-39981 (1997). 